"Buscar la verdad y afianzar los valores transcendentales", misión de las universidades en su artículo primero, inspirado en los principios humanísticos. Ley de Universidades 8 de septiembre de 1970.
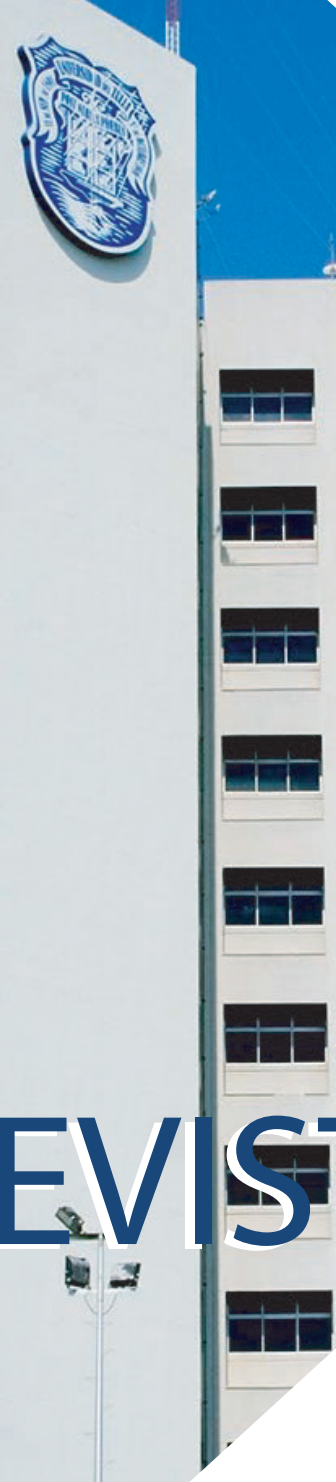
Rev. Téc. Ing. Univ. Zulia. Vol. 43, No. 3, 2020, 122-133

\title{
Arreglo Inteligente de Concentración Solar FV para MPPT usando Tecnología FPGA
}

\author{
Sandoval-Ruiz, Cecilia E. $\mathbb{D}$ \\ Facultad de Ingeniería, Universidad de Carabobo, Venezuela. cesandova@gmail.com \\ https://doi.org/10.22209/rt.v43n3a02 \\ Recepción: 10/02/2020 | Aceptación: 29/06/2020 | Publicación: 01/09/2020
}

\begin{abstract}
Resumen
La presente investigación comprende el estudio de los sistemas fotovoltaicos y las actuales técnicas para el seguimiento del punto de máxima potencia, en relación a la irradiancia y temperatura, a fin de proponer un esquema FV reconfigurable, la simplificación del número de componentes actuadores en el arreglo de seguimiento solar y circuitos de recuperación de calor regenerativo, aplicando control neuronal en la adaptación de los coeficientes de eficiencia de las etapas del sistema. El método consiste en identificar la correspondencia de los SFV con la arquitectura circuital LFSR y la incorporación de coeficientes adaptativos, de forma de generalizar el modelo de optimización sobre una ANN parametrizada. Se realizó la descripción en VHDL de la arquitectura para su síntesis sobre tecnología FPGA, a fin de tratar de manera eficiente la complejidad computacional, procesamiento paralelo y factibilidad técnica-ambiental del diseño. Entre los resultados se presenta una técnica alernativa, basada en circuitos auto-similares, con etapas de ganancia adaptativa, almacenamiento y realimentación configurable. Esta investigación cond uce al concepto de sistemas inteligentes de concentración, el cual aporta un modelo valioso, que puede ser aplicado en el área de ingenieria, diseño e investigación científica.
\end{abstract}

Palabras Clave: sistemas fotovoltaico; concentradores ópticos; seguimiento de punto de máxima potencia; arreglo de compuertas programables por campo FPGA; modelo LFSR.

\section{Smart PV Solar Concentration Arrays for MPPT using FPGA Technology}

\begin{abstract}
The present research includes the study of photovoltaic systems and current techniques for maximum power point tracking, in relation to irradiance and temperature, a flap of proposing a reconfigurable PV scheme, simplifying the number of actuator components in the arrangement solar monitoring and regenerative heat recovery circuits, applying neural control in the adaptation of the coefficients of efficiency of the stages of the system. The method consists of identifying the correspondence of the SFVs with the LFSR circuit architecture and the identification of adaptive coefficients, in order to generalize the optimization model on a parameterized ANN. The VHDL description of the architecture was made for its synthesis on FPGA technology, in order to efficiently deal with the computational complexity, parallel processing and technical-environmental feasibility of the design. The results present an alternative technique based on self-similar circuits, with stages of adaptive gain, storage and configurable feedback. This research leads to the concept of intelligent concentration systems, which provides a valuable model, which can be applied in the area of engineering, design and scientific research.
\end{abstract}

Keywords: photovoltaic systems; optical concentrators; maximum power point tracking; programmable gate arrangement by FPGA field; LFSR model. 


\section{Introducción}

Dada la importancia de optimizar los sistemas de energías renovables no convencionales (ERNC), en relación a la densidad de energía de los convertidores y la eficiencia de los componentes, se presentan diversas investigaciones en este campo, que giran alrededor de los esquemas de control para seguimiento del punto de máxima potencia (MPPT), a través de inteligencia artificial, optimización de enjambre de partículas y otros métodos híbridos [1]. En esta investigación se han revisado los métodos basados en redes neuronales artificiales (ANN), arreglos con estructura Linear Feedback Shift Register (LFSR) aplicados en energías renovables [2-9] y algoritmos adaptativos [10].

El estudio parte del comportamiento del sistema, a fin de detectar vacíos tecnológicos en la generalización de estrategias de optimización a partir del modelo. Analizando la dinámica de las fuentes de ERNC (por su intermitencia), presentan desafios para su control [2-3], como capacidad de cómputo y procesamiento concurrente, donde la tecnología Field Programmable Gate Array (FPGA), se perfila como una alternativa de solución para implementar, de manera eficiente, sistemas embebidos en lenguaje descriptor de hardware (VHDL), que permita soportar el entrenamiento en circuito y la adaptación dinámica.

En el caso de los sistemas fotovoltaicos (SFV) es posible la optimización de etapas funcionales, en término de variables independientes, tal es el caso de la irradiancia, potencia recibida por unidad de superficie, y temperatura de los módulos, dando lugar a combinaciones de métodos de optimización [11-12]. En esta área se ha detectado la necesidad de un modelo matemático que incorpore los parámetros del sistema, describa los componentes y su comportamiento, con el objetivo de definir el control dinámico aplicando coeficientes adaptativos, a partir de la identificación de correspondencia entre cada una de los subsistemas.

La importancia del método propuesto viene dada por una generalización estructural para reconfiguración inteligente y optimización dinámica de los coeficientes que inciden en las variables del modelo, a través de una ANN sobre FPGA [13-14], con arquitectura LFSR. Así como el diseño en hardware del método numérico de MPPT del SFV, a través de la sincronización entre las técnicas de optimización pasivas: interconexión del arreglo, concentración solar, que aumentan la eficiencia del panel FV [15], y técnicas activas: MPPT distribuido, inversores multinivel, reconfiguración dinámica (arreglo de relés), relocalización, recableado eléctrico, mediante la extrapolación de tecnología de hardware reconfigurable al sistema de potencia. Adicional a técnicas innovadoras: arreglos ópticos configurables [12] y modificación espectral de irradiancia (IR-mod).
Comunmente, el sistema de seguimiento modifica la posición (de los ejes $x, y$ ) de los paneles del arreglo FV [16], a fin de obtener la mayor radiación solar incidente en su superficie. Sin embargo, este esquema incorpora un conjunto de motores y elementos móviles al arreglo que aumentan su complejidad y fallas probables del sistema. Por lo que se propone el estudio de alternativas, para mejorar las condiciones de irradiancia, a fin de extender el número de horas de sol pico (HSP), dejando fija la posición de los paneles de la estación terrena (disminuyendo el número de componentes), cuya función será el direccionamiento de la radiación solar, hacia el arreglo fotovoltaico distribuido. Todo esto sincronizado con el MPPT e incorporación de almacenamiento térmico y almacenamiento transitorio en ultra-condensadores (considerando el tiempo útil de baterías respecto a los paneles FV), recuperadores de calor regenerativo (RCR) en los paneles, para aumentar su eficiencia.

En primer lugar se estudian los componentes del SFV para identificar los modelos por etapas.

Arreglo óptico, este corresponde a la configuración geométrica de lentes ópticas, mecanismos de direccionamiento, concentradores solares luminiscentes (LSC) [17], guía de onda (fibra óptica), concentradores fotovolaticos (CPV) [18], etc., que pueden ser diseñados de manera dedicada para cada elemento de conversión, multiplexados en el espacio, o aplicando proyección dinámica [12]. Sus componentes definen una ganancia, dada por los coeficientes específicos del modelo (fijos o adaptativos) sobre la señal de entrada, es decir, el efecto del dispositivo óptico en la trayectoria y magnitud de la radiación solar incidente.

Se propone un arreglo adaptativo, en correspondencia con el concepto de antenas inteligentes [10], que incorporen lentes con función selectiva en dos etapas: direccionamiento heliostático y concentración en el panel, sub-celdas (configuración tándem), filtrado por componente espectral de radiación, expresada de forma matemática en un rango específico para los aportes de energía (ver Tabla 1), que busca reducir el material FV y aumentar la eficiencia. En su diseño se ha identificado la correspondencia del modelo con la estructura LFSR en los elementos: aportes de ganancia adaptativa, almacenamiento transitorio (un tiempo $\tau$ ) y realimentación de la radiación, dado por la reflexión en el material.

En una primera definición se especifican los coeficientes: $w_{U V}$ ultravioleta, $w_{F V}$ luz visible, $w_{I R}$ infrarrojo, por rango $\lambda$, que luego pueden ser generalizados en el modelo integrado, según cada uno de los componentes de la irradiancia recibida $x_{i}$, para función de concentración / atenuación / captación.

Arreglo Fotovoltaico, comprende los elementos de la unidad de generación de energía FV. Este arreglo puede ser reconfigurado durante la operación [19], para 
Tabla 1. Energía Solar y Almacenamiento

\begin{tabular}{|c|c|c|c|c|}
\hline Radiación & Tecnología & Almacenamiento Directo & Fórmula & Longitud de Onda \\
\hline \multirow[t]{2}{*}{$\begin{array}{l}\text { Ultravioleta } \\
10,49 \%\end{array}$} & Esterilización, UV-C & $\begin{array}{l}\text { Conservación biomasa y } \\
\text { tratamiento post-cosecha }\end{array}$ & $w_{U V} \cdot x_{i}+b_{i}$ & $10 \mathrm{~nm} \leq \lambda \leq 400 \mathrm{~nm}$ \\
\hline & Fotovoltaica & Condensadores / Baterías & \multirow{4}{*}{$w_{F V} \cdot x_{i}+b_{i}$} & \multirow{4}{*}{$401 \mathrm{~nm} \leq \lambda \leq 750 \mathrm{~nm}$} \\
\hline \multirow{3}{*}{$\begin{array}{c}\text { Fotones } \\
\text { Luz Visible } \\
42,74 \%\end{array}$} & Baterías fotoeléctricas & $\begin{array}{l}\text { Separación de compuestos } \\
\text { químicos fotoactivos }\end{array}$ & & \\
\hline & Fotosíntesis química & Plantas industriales (algas) & & \\
\hline & Síntesis en FVPGA & HW program. por fotones & & \\
\hline $\begin{array}{l}\text { Calor (I.R) } \\
46,77 \%\end{array}$ & $\begin{array}{c}\text { Termo-Solar } \\
\text { Termo-Eléctrico }\end{array}$ & $\begin{array}{l}\text { Sales de almacenamiento } \\
\text { térmico }\end{array}$ & $w_{i R} \cdot x_{i}+b_{i}$ & $751 \mathrm{~nm} \leq \lambda \leq 4000 \mathrm{~nm}$ \\
\hline
\end{tabular}

minimizar las pérdidas por efecto Joule y el efecto de paneles con fallas específicas. En esta etapa se requiere optimizar la conversión fotoeléctrica, a partir de la configuración del arreglo y electrónica de control. El arreglo FV conecta $n_{p}$ módulos en configuración serie / paralelo, para el maximun power point (MPP) del arreglo y los módulos, por la naturaleza dinámica de este parámetro, en función de las condiciones de los paneles fotovoltaicos en un momento específico, se debe definir una matriz de conmutación del arreglo (MCA). Los módulos fotovoltaico, serán los responsables de definir la capacidad del proceso de conversión de la radiación incidente a energía eléctrica, lo cual está asociado al material fotoeléctrico, la configuración de las celdas y propiedades de panel, a nivel de tecnología [20].

Arreglo deElectrónica de Potencia, está compuesto por los optimizadores electrónicos, convertidores DCDC, almacenamiento transitorio en ultra-condensadores, realimentación de control para MPPT y los módulos inversores. Los módulos electrónicos, comprenden los elementos de control e interruptores controlados, definiendo los niveles de la salida del sistema, a valores óptimos, aplicando un algoritmo basado en un único MPP, que depende de factores externos de temperatura e irradiancia, como se muestra en la Figura 1.

En las curvas se observa la relación entre las variables independientes: irradiancia y temperatura respecto a los parámetros eléctricos del panel fotovoltaico: potencia, corriente y voltaje, lo que representa un insumo para el entrenamiento de la ANN de optimización.

Arreglos de Realimentación de Energía, estos comprenden subsistemas de recuperación de energía regenerativa, donde se plantea el modelo realimentado para fotones no convertidos, aplicando tecnología PERC, bifacial, etc., que permitan recolectar la radiación reflejada, recuperadores de calor residual, ventilización forzada, refrigeración de paneles $\mathrm{FV}$, para colectores térmicos y conversión usando material termoeléctrico, a fin de optimizar el aprovechamiento de energía del SFV. La energía residual realimentada puede ser manejada a través de conceptos como cosechamiento de energía para alimentar dispositivos electrónicos, aplicable para

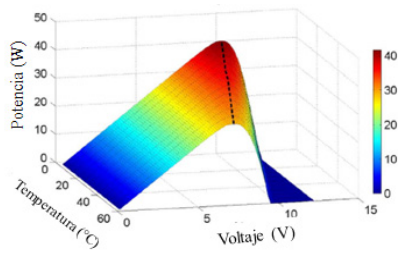

(a) Temperatura sobre P-V

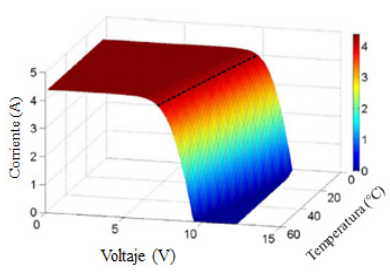

(c) Temperatura sobre I-V

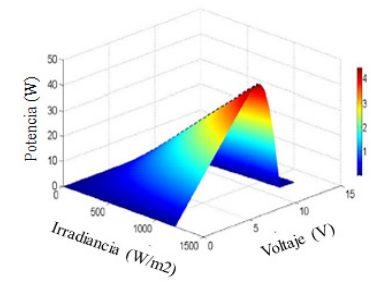

(b) Irradiancia sobre P-V

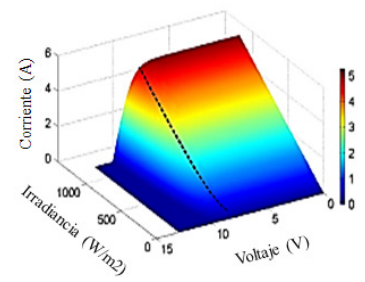

(d) Irradiancia sobre I-V
Figura 1. Efecto de condiciones ambientales en conversión fotovoltaica [21]

el suministro de la etapa de optimización, manteniendo como criterio la sostenibilidad y bajo impacto ambiental. De esta manera, encontramos un modelo del SFV que coincide con la estructura de las etapas, donde se presentan ponderación de aportes, almacenamiento intermedio de energía y realimentación selectiva por componentes de energía, dando lugar a la ecuación descriptiva parametrizable.

\section{Desarrollo Conceptual del Modelo LFSR para Siste- mas Fotovoltaicos}

Se propone la incorporando un sistema satélite (conformado por uno o más reflectores ópticos), así como la optimización de parámetros (dependientes de características topográficas y condiciones climáticas): número de HSP sobre la superficie distribuida del arreglo fotovoltaico, ángulo de incidencia óptima, MPPT y control de temperatura (ver Figura 2). 


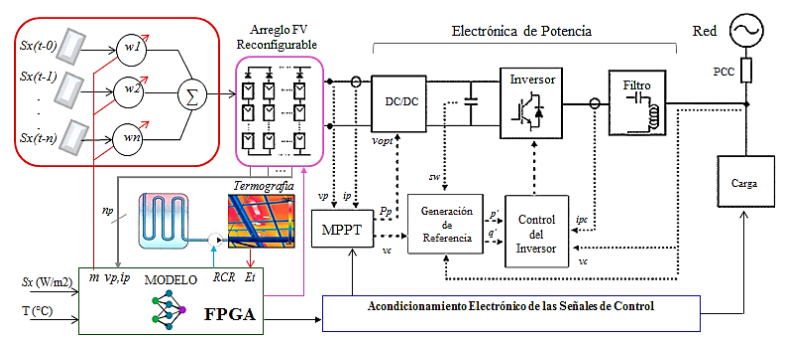

Figura 2. Esquema Conceptual de Actualización del control MPPT en SFV

La técnica propuesta se basa en una ANN para el modelado del SFV y adaptación de los coeficientes a través del algoritmo de optimización, implementado sobre un FPGA. A partir de las entradas/salidas del sistema: irradiancia $S_{X}\left(S_{U V}, S_{L V}, S_{I R}\right)$, corriente en el punto de máxima potencia $\mathrm{I}_{\mathrm{M}} \mathrm{PP}$, voltaje MPP $\mathrm{V}_{\mathrm{MPP}}$, temperatura del panel $\mathrm{T}_{\mathrm{p}} \mathrm{y}$ energía térmica $\mathrm{ET}$, aplicando redes neuronales convolucionales (CNN) [14] de imágenes termográfica del arreglo FV, para el control del sistema centralizado de seguimiento (driver_motores), control de temperatura de los paneles (ventilación forzada), recuperadores de calor residual (RCR), configuración (relés), ganancia adaptativa, concentración y MPPT aplicando modulación por ancho de pulso (PWM).

En [19] se presenta el estudio detallado de los factores que influyen en el rendimiento del arreglo fotovoltaico. Los métodos varían en su complejidad, sensores necesarios, velocidad de convergencia, efectividad, etc. en relación a la dinámica del sistema, requiriendo métodos avanzados MPPT (ver Tabla 2), el cual se hace variando el valor de la carga de forma que el SFV pueda entregar la mayor potencia posible.
Esta variación de carga es controlada por un convertidor DC-DC, el cual tiene la característica de elevar o reducir una tensión modificando el ciclo de trabajo y la carga equivalente del circuito, logrando una tal que consuma la potencia máxima del panel. Donde se genera una señal PWM, como señal de control del interruptor, esta señal es sincronizada con el MPP, para la carga del condensador.

Para el MPP, donde se relacionan parámetros como la irradiancia, temperatura y carga equivalente el generador fotovoltaico, se requiere un algoritmo de seguimiento [11], [19]. La reconfiguración dinámica permite mejorar el rendimiento del arreglo fotovoltaico, a través de la reducción de las pérdidas Joule. Es así como, dependiendo de la condiciones ambientales, la conexión o desconexión de paneles se haría mediante interruptores. En la etapa de optimización uno de los puntos de interés es la electrónica de potencia responsable del acoplamiento del generador fotovoltaico a la carga. El convertidor DC-DC elevador puede ser controlado por corriente, tensión, basados en el ciclo útil de operación o por control magnético MC, basado en inductancia variable.

\section{Metodología}

Para el diseño del modelo de optimización basado en hardware se plantean criterios de sostenibilidad, en tal sentido, se deben diseñar sistemas regenerativos, realimentados con aporte en el presupuesto de energía, ciclos de reutilización, reciclaje, reconfiguración dinámica y realimentación de subproductos o energía, en correspondencia con el modelo circular. Para ello se realiza en la primera etapa del procedimiento de investigación un análisis cualitativo y cuantitativo de los métodos de optimización por etapa, resumidos en la Tabla 3.

Tabla 2. Estudio de Métodos MPPT

\begin{tabular}{cccc}
\hline Tecnología de Control MPPT & Descripción Técnica / Arreglo Fotovoltaico & Concentrador & Ref. \\
\hline FPGA - Control & Estudio de métodos usando FPGA & - & En el panel \\
ANN & High Concentrator Photovoltaic (HCPV) & - & {$[1]$} \\
Lógica Difusa & Algoritmo RTRL, O\&P, Híbridos & - & {$[23-28]$} \\
Control Centralizado & Controlador de tres etapas / FPGA & - & {$[29-30]$} \\
ANFIS - FPGA, reconfig. & Módulos fotovoltaicos distribuidos & - & {$[31]$} \\
\hline
\end{tabular}


Tabla 3. Optimización de Etapas del SFV




A partir de las estimaciones obtenidas en el estudio de las configuraciones del arreglos de seguimiento: fijos, seguimiento en eje horizontal (HSAT), en eje vertical (VSAT) o en dos ejes (HVSAT), aplicando un estimador [40], se observa el aporte del seguimiento en la estación terrena, a un costo de implementación del conjunto de motores de accionamiento para el posicionamiento de los paneles en relación al peso de la estructura móvil, en la matriz de $n_{p}$ elementos. Por lo que se plantea una simplificación del SFV sin disminuir su eficiencia, a través de un adaptador, aplicando la propiedad conmutativa entre etapas, que direccione la radiación a cierta altura, con ángulo óptimo de incidencia sobre el arreglo fotovoltaico fijo.

Se definieron las estrategias para el control de los factores a optimizar en los SFV e identificación de los términos del modelo matemático (ver Tabla 4).
El modelado se realizó a través de la descripción del comportamiento por funciones del SFV, la ANN puede realizar ajustes en el modelo matemático, a través de identificación de configuración y parámetros, a partir del balance de energía:

$$
E_{c}(n)=w_{c}\left(w_{F V} \cdot S_{x}(n)\right)+b_{s} \cdot E_{F V}(n-1)+w_{R C R} \cdot E_{T}(n-1)(1
$$

Donde $w_{c}$ es un coeficiente asociado al modelo de optimización (concentración, ángulo de incidencia), $w_{F V}$ el coeficiente de conversión del panel FV (propio de la tecnología del SFV), $b_{s}$ habilitación selectiva, $E_{c}(n-1)$ la energía realimentada: $E_{F}(n-1)$ energía reflejada y $E_{T}(n-$ 1) energía térmica residual, con $w_{R C R}$ como ganancia de realimentación al sistema de conversión. Los efectos térmicos pueden ser compensados: $w_{T}\left(1+T C_{I S C} \cdot \Delta T\right) \cdot I_{F V}$, con la corriente proporcional a la conversión de irradiancia, siendo $w T$ coeficiente de optimización térmica. De estos términos se identifican las señales de control de actuadores (ver Tabla 5).

Tabla 4. Factores de optimización del SFV

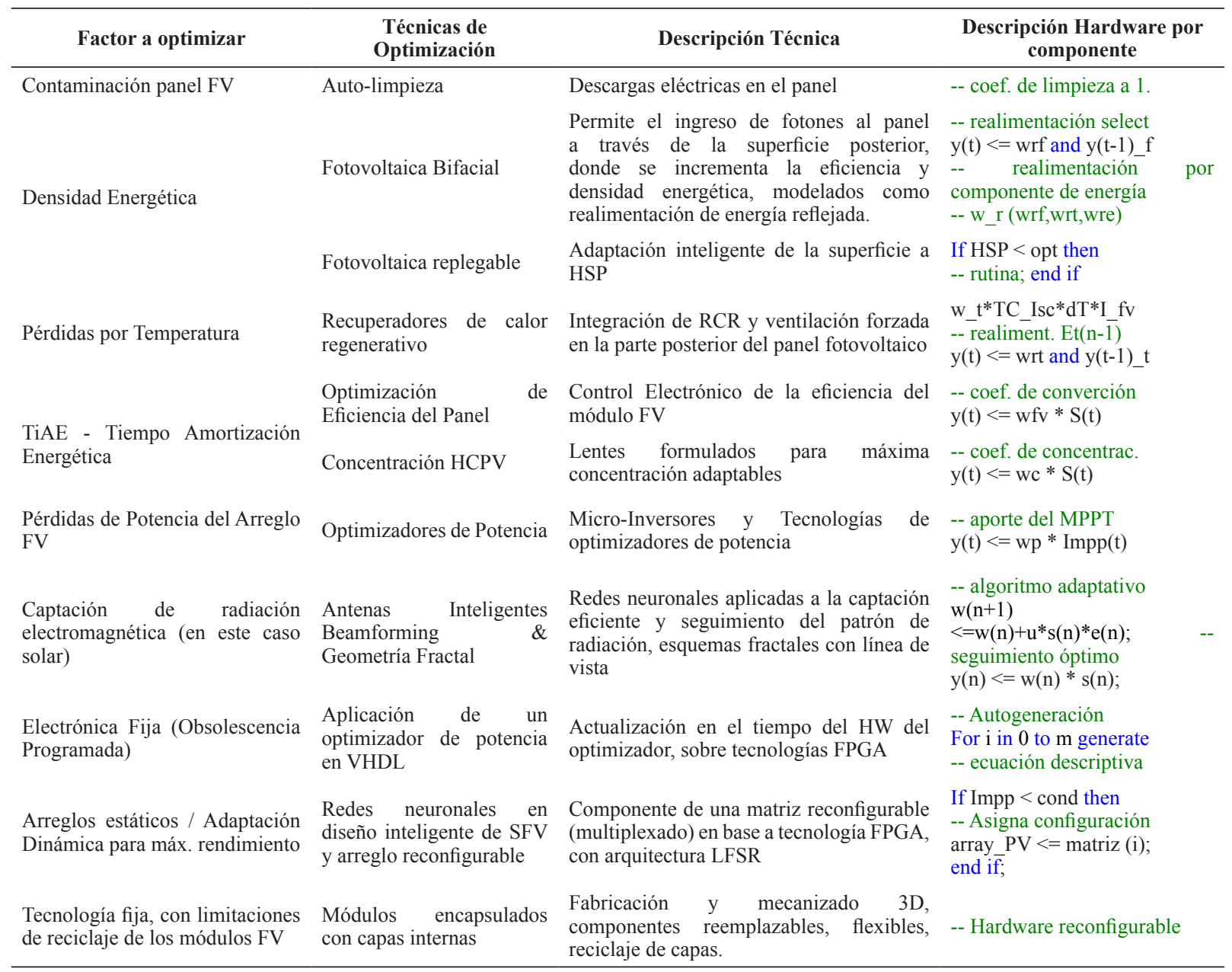


Tabla 5. Descripción de las etapas de control de actuadores del SFV

\begin{tabular}{|c|c|}
\hline Aplicación & Códigos de la Descripción del Hardware en VHDL \\
\hline Control de Refrigeración & $\begin{array}{l}\text {-- Activación de la bomba de circulación de refrigerante en el arreglo FV. } \\
\text { If t_ref }<\text { ANN_RCR then } \\
\text { Bomba }<=\text { ' } 1 \text { '; else Bomba }<=\text { ' } 0 \text { '; end if; } \\
\text {-- Implementación del recuperador de calor residual RCR. }\end{array}$ \\
\hline Algoritmo MPPT & 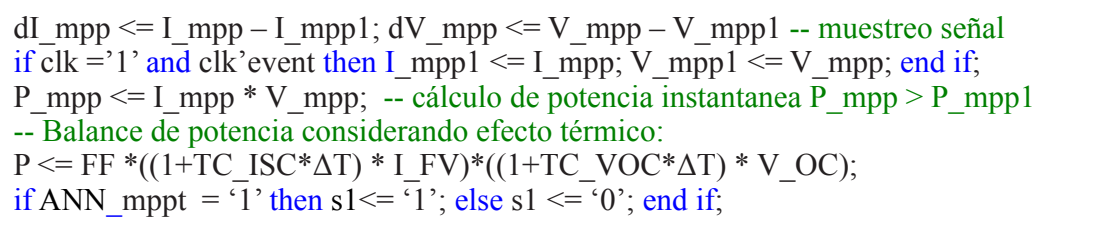 \\
\hline Control Accionamientos & $\begin{array}{l}\text {-- ANN_SG define el sentido de giro del motor de paso de seguimiento solar mecánico. } \\
\text { If ANN_SGM1= '0' then -- define el sentido de giro del motor de paso M1 } \\
\text { For i in } 1 \text { to } 4 \text { loop driver_m1 } 1 \text { (i-1) }<=\text { '0'; driver_m } 1 \text { (i) }<=\text { ' } 1 \text { ' after } 10 \mathrm{~ms} \text {; end loop; } \\
\text { else } \\
\text { For i in } 4 \text { downto } 1 \text { loop driver_m1 } 1(\mathrm{i}+1)<=\text { ' } 0 \text { '; driver_ml } 1 \text { (i) }<=\text { ' } 1 \text { ' after } 10 \mathrm{~ms} \text {; } \\
\text { end loop; end if; -- se definió con la ec. del modelo el accionamiento selectivo LFSR } \\
--\sum w i * x i-->G F(m): y n<=w(i) \text { and } x(i) \text { or } w(i-1) \text { and } x(i) \ldots x o r y(i-1)\end{array}$ \\
\hline
\end{tabular}

De esta forma, el control se establece a partir de las salidas de la ANN, combinaciones de relés de configuración de las conexiones del arreglo, RCR, MPPT de cada panel del arreglo, señales de control de los motores del mecanismo de seguimiento solar (altura, ángulos de elevación y azimut, complementarios al ángulo de incidencia del arreglo FV, entre otros). Una vez seleccionado el método de optimización por modelado dinámico, se aplican las técnicas de descripción del hardware para manejo de los coeficientes de optimización y la descripción de comportamiento en VHDL de los circuitos. De esta manera, se estandariza la estructura matemática para las descripciones sistematizadas de la ANN optimizadora. Finalmente, la síntesis del dispositivos electrónico inteligente (IED).

\section{Resultados y Discusión}

Del análisis de los métodos, complejidad de implementación y factorización de los coeficientes de optimización se modeló el sistema de seguimiento heliostático, con línea de vista hacia el arreglo FV, para direccionamiento de la radiación, para una longitud de onda selectiva y filtrar la radiación UV con el fin de proteger la superficie de este tipo de ondas, lo cual puede tener amplia aplicación en la protección de fauna, glaciares y ambientes forestales. De manera de establecer parámetros óptimos de altura, concentración y ángulos de incidencia / reflexión, que serán adaptados por la ANN. Este arreglo inteligente, permiten simplificar el módulo de seguimiento solar (reducción del número de actuadores en el arreglo), representando una innovación tecnológica. La técnica propuesta está basada en el modelo desarrollado (ver Tabla 6) y comprende la adaptación de coeficientes sobre el modelo de balance de energía.

Todo esto a fin de obtener máxima eficiencia: en la etapa de concentración espectral de luz visible, etapa de conversión, etapa de electrónica de potencia, cosechamiento de energía y almacenamiento, de forma conjunta en el SFV. Partiendo de la identificación de una estructura común para las etapas estudiadas, se identifica un sistema regenerativo que comprende almacenamiento y realimentación, así como una etapa de control híbrido que combina MPPT con adaptación de coeficientes del modelo. Así, se halla una ecuación generatriz para la descripción geométrica (configuración de arquitectura) y parámetrica, usando la herramienta de síntesis para VHDL [41] y la instanciación de los componentes neuronales (ver Tabla 7).

En la descripción VHDL se ha parametrizado la ANN, donde los pesos sinápticos corresponden a los coeficientes de optimización del modelo desarrollado. Los parámetros de configuración (cantidad de neuronas, capas, tasa de aprendizaje, pesos sinápticos) se establecen según el sistema, los criterios de optimización ambientales y targets de seguimiento (en base a un conjunto de entrenamiento, validación y prueba), para la adaptación de la red ANN sobre hardware [5], conectado en línea con el SFV. 
Tabla 6. Características Técnicas del Modelo Optimizador

\begin{tabular}{|c|c|c|}
\hline Características Técnicas & Logros de Innovación & Motivos para su implementación \\
\hline Orientado a Hardware & Sintetizable sobre tecnología FPGA & Portabilidad del diseño \\
\hline $\begin{array}{l}\text { Modelo multi-etapa (parame- } \\
\text { trizable) }\end{array}$ & $\begin{array}{l}\text { Se incluyen las variables de las diversas etapas } \\
\text { para el MPPT }\end{array}$ & Se definen coeficientes de optimización en cada etapa \\
\hline Ecuación generatriz & Sistematización de la descripción & Parametrizable para sistemas particulares \\
\hline Conmutabilidad & $\begin{array}{l}\text { Simplificación de componentes y elementos } \\
\text { actuadores }\end{array}$ & $\begin{array}{l}\text { Manejo de términos por factor de incidencia sobre el } \\
\text { arreglo. }\end{array}$ \\
\hline Escalabilidad & $\begin{array}{l}\text { Capacidad para incluir módulos con la misma } \\
\text { estructura LFSR }\end{array}$ & Se pueden implementar optimizaciones at custom \\
\hline Flexibilidad & $\begin{array}{l}\text { Se describen componentes actualizables y re- } \\
\text { utilizables. }\end{array}$ & $\begin{array}{l}\text { Aumento del tiempo de vigencia de los diseños en } \\
\text { hardware }\end{array}$ \\
\hline Reconfiguración dinámica & $\begin{array}{l}\text { Modular / diferencial para actualización de } \\
\text { etapas }\end{array}$ & $\begin{array}{l}\text { Soporte de SFV distribuidos y extrapolación del mod- } \\
\text { elo }\end{array}$ \\
\hline ANN-MPPT & $\begin{array}{l}\text { Adaptación de coeficientes del modelo (con- } \\
\text { siderando las curvas de eficiencia del panel) }\end{array}$ & $\begin{array}{l}\text { La alta demanda de cómputo del control en ERNC por } \\
\text { la dinámica del sistema, requiere la optimización del } \\
\text { algoritmo secuencial perturba y observa para MPPT }\end{array}$ \\
\hline Correspondencia & \multicolumn{2}{|c|}{$\begin{array}{l}\text { Identificación de estructura LFSR en el SFV y optimizador neuronal, donde los pesos de la ANN tienen } \\
\text { significado físico en los coeficientes de optimización. }\end{array}$} \\
\hline
\end{tabular}

Tabla 7. Descripción del modelo ANN parametrizado

Códigos de la Descripción del Hardware ANN en VHDL

Entrenamiento de la ANN

\begin{tabular}{|c|c|c|c|c|}
\hline \multicolumn{4}{|c|}{ 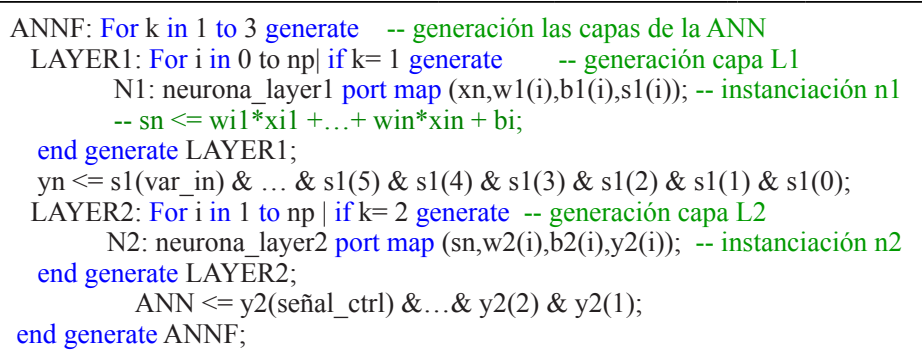 } & $\begin{array}{l}\text { type matriz_peso is array of (m downto } 0) \text { of } \\
\text { std_logic_vector }(7 \text { downto } 0) \text {; } \\
-- \text { Adaptación de la matriz de pesos por } \\
\text { iteración: } \\
\text { if clk'event and clk ='1' then } \\
\mathrm{e}(\mathrm{n})<=\mathrm{t}(\mathrm{n})-\mathrm{y}(\mathrm{n}) \text {; } \\
\text { wi(n+1) } \\
<=\text { wi(n) }+\mathrm{u}^{*} \operatorname{xi}(\mathrm{n})^{*} \mathrm{e}(\mathrm{n}) \text {; }\end{array}$ \\
\hline Circuito & Slices & 4-LUTS & \multicolumn{2}{|c|}{ Factor $\cdot$ Unidades_parametrizadas } \\
\hline $\mathrm{ANN}\left(v a r \_i n, n p, s e \tilde{n} a l \_c t r l\right)$ & 100 & 198 & \multicolumn{2}{|c|}{ n_layer (neurona $\cdot$ pesos_sinápticos $\cdot \mathrm{m}$ bits) } \\
\hline Algoritmo Adaptativo & 80 & 148 & \multicolumn{2}{|c|}{$(\mathrm{n} 1 \cdot$ var_in $+\mathrm{n} 2 \cdot$ señal_ctrl $) \cdot \mathrm{m}$ bits } \\
\hline \multicolumn{5}{|l|}{ Expresión descriptiva: } \\
\hline \multicolumn{5}{|c|}{ Capa 2,3 / Optimización Capa 1 / Modelado del SFV } \\
\hline
\end{tabular}


En una configuración específica del SFV se ha incorporado el arreglo óptico inteligente para el aprovechamiento máximo de la radiación incidente $S_{x^{\prime}}$ con coeficientes wadap [42] control de temperatura y RCR para recuperación de energía residual de los paneles:

$$
y(t)=\sum_{k=1}^{m} w_{C S}(k) * \sum_{j=1}^{n p} w_{M P P}(j) * \sum_{i=1}^{n p} w_{c}(i) * w_{F V}(i) * x(t)+w_{R} * y(t-1)
$$

Con $w_{C S}$ ganancia del seguidor (para los $m$ elementos del arreglo propueso), $w_{M P P}$ ganancia dinámica del optimizador: MPPT, control de temperatura, etc., $x(t)$ irradiancia total, $w_{R}$ habilitador de realimentación de los componentes de energía residual $y(t-1)$. Destacando que un operador puede ser un circuito LFSR con la función concatenada en su estructura:

$$
y(t)=f_{\text {OPT }}\left(f_{F V}(x(t))\right)+w_{R} * y(t-1), \operatorname{con} f_{F V}=\sum_{i=1}^{n p} w_{f}(i) * x_{f}(i)+E_{f}(t-1)
$$

Se obtiene como resultado el modelo de optimización, basado en el esquema LFSR, que permite la configuración dinámica y descripción del control inteligente sobre dispositivos FPGA. Adicionalmente, se incluyen selectores (relés) para las ramas de operadores por arrays, por lo que el coeficiente de una rama define el peso correspondiente o la selección ON/OFF, en el caso de ser un operador LFSR (ver Figura 3).

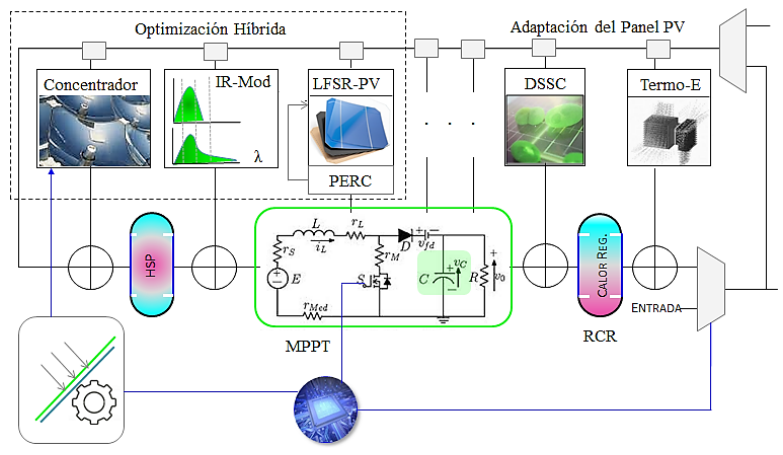

Figura 3. Modelo LFSR de optimización por arreglo de concentración solar
El modelo LFSR-FV está pensado para formular la configuración: fotoeléctrica, termoeléctrica, usando optimización ANN. Igualmente, se establece la correspondencia con módulos funcionales reconfigurables (ver Tabla 8), para establecer las propiedades del concentrador CSL, etapas del SFV y términos de realimentación (eléctrica, térmica, fotónica)

La identificación de correspondencia entre los modelos, coeficientes del SFV (primera capa) y coeficientes de optimización (capas profundas) de la ANN [5], permite el ajuste de la respuesta de eficiencia, MPPT del sistema total, y la generación del código en VHDL. Al mismo tiempo que puede aplicarse transferencia de aprendizaje de la ANN [13] para servicio de modelado y ajuste fino para la optimización remota del sistema fotovoltaico.

En el contexto de la actual disminución del 82\% de los costos de la energía FV [43], resulta de interés la optimización espectral de radiación directa, difusa y reflejada [44] como aportes parciales en el modelo. Los resultados alcanzados pueden ser extrapolados en en MPPT eólica [45], ERNC [2], sistemas regenerativos en ingeniería, formulación ANN sobre hardware [46] para sensibilización FV, arreglos ópticos, modelado térmico de paneles bifaciales [47], refrigeración de paneles para MPP FV/T [48], optimizadores de potencia [49] y sistema híbridos de generación eléctrica [50], eficiencia energética [51] y desarrollo de tecnologías de minimo impacto ambiental [52-53].

\section{Conclusiones}

Gracias al modelo de optimización sobre arreglos de concentración solar FV para MPPT, basada en ANN, arquitectura LFSR y algoritmos adaptativos responsables del ajuste de los coeficientes del sistema, se ha logrado dotar de inteligencia al SFV, aplicando circuitos de procesamiento paralelo y reconfiguración dinámica del arreglo, lo que introduce una mejora en el aprovechamiento de las condiciones de irradiancia, HSP, modificación espectral, control de temperatura de los módulos FV, realimentación de componentes de energía residual, para elevar el rendimiento total, a través de la

Tabla 8. Correspondencia del Modelos LFSR

\begin{tabular}{llll}
\hline \multicolumn{1}{c}{ LFSR } & \multicolumn{1}{c}{ Capas de operación } & \multicolumn{1}{c}{ Almacenamiento } & \multicolumn{1}{c}{ Realimentación } \\
\hline $\begin{array}{l}\text { Fractal } \\
\text { ANN }\end{array}$ & Neuro-Operadores & TDL & $y(t-1)$ \\
Óptica & Ganancia Concentrador & Luminiscencia (Shift Stokes) & Reflexión de fotones \\
Fotovoltaica & Tándem PV & Shift corriente / Calor Residual & PERC / $\lambda$ de irradiancia \\
Electrónica & DSP / PWM & Ultra-condensadores UC & Recuperador Calor Reg. \\
Smart Grid & IEDs- Convertidores & Almacenamiento de Energía & E. Reversible \\
$\begin{array}{l}\text { Modelo } \\
\text { CSR }\end{array}$ & COD / RCR & $\tau$ (retardo de radiación) & Coeficientes de optimización \\
\hline
\end{tabular}


contribución de cada etapa.

De esta manera, se avanza en el concepto de Fotovoltaica 2.0, en el marco de la actualización dinámica del hardware e incorporando técnicas de digitalización, energy harvesting, arreglos inteligente, HCPV y modularidad, usando tecnología FPGA, con importantes ventajas en el rendimiento y flexibilidad, en comparación con sistemas fijos que requieren mayor intervención en procesos de actualización o migración.

Igualmente, el modelo de optimización desarrollado presenta aportes significativos en la identificación de los parámetros físicos del modelo, almacenamiento y realimentación selectiva, dada su correspondencia con la arquitectura LFSR, lo que permite el ajuste de los coeficientes del sistema aplicando algoritmos adaptativos convencionales o diseños de aproximaciones orientados a hardware, para manejo inteligente del aporte de energía solar, así como de factores no contemplados inicialmente en la configuración del modelo ANN.

Observando los reportes de eficiencia del mecanismo de seguimiento y combinación de métodos, se planteó el direccionamiento de la energía solar hacia el arreglo fotovoltaico fijo, en combinación con la optimización. Así, los resultados alcanzados dan lugar a nuevos espacios de desarrollo y técnicas competitivas en aplicaciones de smart grid y centrales virtuales de energía.

Siendo importante la consideración en los conceptos de diseño de aspectos tales como factibilidad socio-ambiental, en los que se incorpore la actualización del talento humano en tecnología de energías renovables, tele-configuración, nuevas áreas de desarrollo sostenible, eco-responsabilidad con la fauna, hábitat, materiales y recursos naturales, aprovechamiento óptimo de la infraestructura (para reconversión de unidades de producción de energía convencional), tecnología de hardware reconfigurable para la adaptación / actualización dinámica en el tiempo (reutilizabilidad y reciclaje), eficiencia energética de los dispositivos, a partir del modelo desarrollado.

\section{Referencias Bibligráficas}

[1] Mellit A., Kalogirou S. A. : "MPPT-based artificial intelligence techniques for photovoltaic systems and its implementation into field programmable gate array chips: Review of current status and future perspectives". Energy, Vol. 70, N C, (2014) 1-21.

[2] Sandoval-Ruiz C. : "Modelo VHDL de Control Neuronal sobre tecnología FPGA orientado a Aplicaciones Sostenibles". Ingeniare. Rev. chilena de ingeniería, Vol. 27, № 3, (2019) 383-395.

[3] Sandoval-Ruiz C. : "Control de Micro-Redes de Ener- gía Renovable a través de estructuras LFCS Reconfigurables en VHDL". Ciencia y tecnología, Vol. 18, (2018) 71-86.

[4] Sandoval-Ruiz C. : "Códigos Reed Solomon para sistemas distribuidos de energías renovables y smart grids a través de dispositivos electrónicos inteligentes sobre tecnología FPGA". Memoria Investigaciones en Ingeniería, Vol. 16, (2018) 37-54.

5] Sandoval-Ruiz C. : "LFSR-Fractal ANN Model applied in R-IEDs for Smart Energy". IEEE Latin America Transactions, Vol. 18, N 04, (2020) 677-686.

[6] Sandoval-Ruiz C. : "Modelo Neuro-Adaptativo en VHDL, basado en Circuitos NLFSR, para Control de un Sistema Inteligente de Tecnología Sostenible". Rev. Universidad, Cienc. y Tecnol. Vol. 21, $\mathrm{N}^{\circ} 85$, (2017) 140-149.

[7] Sandoval-Ruiz C. : "Métodos Numéricos en Diferencias Finitas para la Estimación de Recursos de Hardware FPGA en arquitecturas $\operatorname{LFSR}(\mathrm{n}, \mathrm{k})$ Fractales". Ingeniería Investigación y Tecnología. Vol. XX, Nº3, (2019) 1-10.

[8] Sandoval-Ruiz C. : "Plataforma de Investigación de Redes Eléctricas Reconfigurables de Energías Renovables aplicando Modelos LFSR". Universidad, Ciencia y Tecnología, Vol. 23, N 95, (2019) 103-115.

[9] Sandoval-Ruiz C. : "Operador matemático LFC(n,k) en campos finitos basado en concatenación fractal para GF(2m) - Extendido". Ciencia e Ingeniería, VOl. $41, \mathrm{~N}^{\circ} 2$, (2020) 197-204.

[10] Castellanos J., Sandoval C., Azpúrua M. : "Implementación sobre FPGA de un Algoritmo LMS para un arreglo de antenas inteligentes". Rev. Téc. Ing. Univ. Zulia, Vol. 37, N³, (2014) 270-278.

[11] Rodríguez-Gallegos, Carlos D., et al. : "Global TechnoEconomic Performance of Bifacial and Tracking Photovoltaic Systems". Joule Vol. 4, (2020) 1-28.

[12] Sandoval-Ruiz C. : "Proyecto Cometa Solar - CS para optimización de sistemas fotovoltaicos". Universidad, Ciencia y Tecnología,, Vol. 24, N 100, (2020) 74-87.

[13] Whatmough P. N., Zhou C., Hansen P., Venkataramanaiah S. K., Seo J. S., Mattina M. : "FixyNN: Efficient hardware for mobile computer vision via transfer learning". Proceedings of the 2 nd SysML Conference (2019).

[14] Shawahna A., Sait S. M., El-Maleh A. : "FPGA-based accelerators of deep learning networks for learning and classification: A review". IEEE Access, 7, (2018) 7823-7859. 
[15] NREL National Renewable Energy Laboratory, Best Research-Cell Efficiency Chart, (2020).

[16] Fernández-Ahumada, L. M., et al. : "A novel backtracking approach for two-axis solar PV tracking plants". Renewable Energy Vol. 145, (2020) 1214-1221.

[17] Parola I. : "High performance fluorescent fiber solar concentrators employing double doped polymer optical fibers". Solar Energy Materials and Solar Cells, Vol. 178, (2018) 20-28.

[18] Angulo, M. : "Desarrollo de un Sistema Fotovoltaico de Concentración para aplicaciones de Co-Generación". Tesis de Maestría, Centro de Investigación en óptica, México (2018).

[19] González de la Fuente, J. M. : “Utilización de ultracondensadores para la mejora de eficiencia de una instalación fotovoltaica". Tesis Doctoral. Universidad de Valladolid (2015).

[20] Sandoval-Ruiz, C. : "Arreglos Fotovoltaicos Inteligentes con Modelo LFSR-Reconfigurable". Revista Ingeniería, Vol. 30, N² 2, (2020) 32-61.

[21] Kermadi, M., Berkouk, E. M. : "Artificial intelligencebased maximum power point tracking controllers for Photovoltaic systems: Comparative study". Renewable and Sustainable Energy Reviews, Vol. 69, (2017) 369-386.

[22] Almonacid, F., Fernández, E. F., Rodrigo, P., PérezHigueras, P. J., Rus-Casas, C. : "Estimating the maximum power of a high concentrator photovoltaic (HCPV) module using an artificial neural network". Energy, Vol. 53, (2013) 165-172.

[23] Viloria-Porto, J., Robles-Algarín, C., Restrepo-Leal, D. : "A novel approach for an MPPT controller based on the ADALINE network trained with the RTRL algorithm". Energies, Vol. 11, № 12, (2018) 3407.

[24] Viloria Porto, J. P. : “Diseño e implementación de un controlador neuronal artificial dinámico para optimizar el algoritmo de perturbación y observación en el seguimiento del punto de máxima potencia de un módulo fotovoltaico". Universidad del Magdalena (2018).

[25] Ahmed, C. C., Cherkaoui, M., Mokhlis, M. : "MPPT Control for Photovoltaic System using hybrid method under variant weather condition". In 2019 International Conference on Wireless Technologies, Embedded and Intelligent Systems - WITS (2019) 1-5.

[26] Salimi, M. : "Artificial Neural-Network-Based
Maximum Power Point Tracking for Photovoltaic Pumping System Using Backstepping Controller". RTUCON (2018) 1-7.

[27] García, B., Carmona, C., Rivera, A., Jesús, M., Aguilera, J. : "A differential evolution proposal for estimating the maximum power delivered by CPV modules under real outdoor conditions". Expert Systems with Applications, Vol. 42, N¹3, (2015) 5452-5462.

[28] Punitha, K., Devaraj, D., Sakthivel, S. : "Artificial neural network based modified incremental conductance algorithm for maximum power point tracking in photovoltaic system under partial shading conditions". Energy, Vol. 62, (2013) 330-340.

[29] Pacheco Bautista, D., Cortés Rito, E., Aguilar Acevedo, F. : "Diseño de un controlador de carga de tres etapas para sistemas fotovoltaicos usando lógica difusa". Ingeniare. Rev. chilena de ingeniería, Vol. $27, \mathrm{~N}^{\circ} 4$, (2019) 540-550.

[30] Youssef, A., El Telbany, M., Zekry, A. : "Reconfigurable generic FPGA implementation of fuzzy logic controller for MPPT of PV systems". Renewable and Sustainable Energy Reviews, Vol. 82, (2018) 13131319.

[31] Moon, S., Yoon, S. G., Park, J. H. : "A new low-cost centralized MPPT controller system for multiply distributed photovoltaic power conditioning modules". IEEE Transactions on Smart Grid, Vol. 6, $\mathrm{N}^{\circ}$ 6, (2015) 2649-2658.

[32] Aldair, A. A., Obed, A. A., Halihal, A. F. : "Design and implementation of ANFIS-reference model controller based MPPT using FPGA for photovoltaic system". Renewable and Sustainable Energy Reviews, Vol. 82, (2018) 2202-2217.

[33] Cabello, S. : "Reconfiguración dinámica del campo solar de una planta fotovoltaica para maximizar la producción de energía". Universidad de Sevilla (2018).

[34] Ramos Hernanz, J. A. : "Optimización de modelos de módulos fotovoltaicos y del control del MPPT". Universidad del País Vasco-EHU (2017).

[35] Hassaine, L... : "Implementación de un control digital de potencia activa y reactiva para inversores". Universidad Carlos III de Madrid (2010).

[36] Daorta, F., Warren, S.: "Concentradores luminiscentes para las aplicaciones fotovoltaicas". Universidad del Callao (2015).

[37] González, G. R., Muñoz, S. O. : “Análisis comparativo de dos técnicas para el transporte vertical de la luz 
natural”. REVISTARQUIS, Vol.7, N¹, (2018).

[38] Fernández-Balbuena, A. : "Sistemas ópticos para concentración, captación y guiado de radiación solar". Universidad Complutense de Madrid (2011).

[39] Castro, J. A., García-Alvarado, R., Aguilar, H., SánchezFriera, P. : "Concentradores Solares Luminiscentes". RChD: creación y pensamiento, Vol.3, N5, (2018).

[40] Solar E., Explorador Solar aplicación fotovoltaica. Universidad de Chile (2020). http://ernc.dgf.uchile. cl:48080/fotovoltaico.

[41] Xilinx. ISE Design Suite 11.1. (2020). https://www. xilinx.com/products/design-tools/ise-design-suite. html.

[42] Sandoval-Ruiz C. : "Adaptive Control in VHDL Applied to a Solar Oven". Rev. Colombiana de Tecnol. de Avanzada Vol. 1, №23, (2014) 142-147.

[43] IRENA, How Falling Costs Make Renewables a Cost-effective Investment, (2020). Recuperado de: https://www.irena.org/newsroom/articles/2020/ Jun/How-Falling-Costs-Make-Renewables-a-Costeffective-Investment.

[44] Gómez Sarduy, Julio Rafael, et al. : "Determinación de la radiación solar directa y difusa en la zona de la costa venezolana a partir de variables meteorológicas". Rev. Téc. Ing. Univ. Zulia, Vol. 38, N 2, (2015) 150-158.

[45] González, L. G., et al. : "Diseño de un emulador para sistemas de conversión de energía eólica". Rev. Téc. Ing. Univ. Zulia, Vol. 38, N² 2, (2015) 159-168.

[46] Sandoval-Ruiz, C., Ruiz-Díaz, E. : “Optimizador de Eco-Productos de origen vegetal aplicando Control Neuronal en VHDL". Revista de Ciencia y Tecnología Agrollanía, Vol. 15, (2018) 58-64.
[47] Bernuy Bahamóndez, C. : "Modelamiento térmico unidimensionaly transiente de una celda fotovoltaica bifacial". Universidad de Chile (2018).

[48] Da Rocha, N. M. M., Brighenti, L., Passos, J., Martins, D. : "Photovoltaic Cell Cooling as a Facilitator for MPPT". IEEE Latin America Transactions, Vol. 17, N ${ }^{\circ}$ 10, (2019) 1569-1577.

[49] De Souza Silva, J. L., Moreira, H. S., de Mesquita, D. B., dos Reis, M. V. G., Villalva, M. G. : "Study of Power Optimizers for grid-connected photovoltaic systems". IEEE Latin America Transactions, Vol. 17, $\mathrm{N}^{\circ} 1,(2019)$ 127-134.

[50] Sandoval-Ruiz, C. : "Diseño conceptual de un módulo híbrido para Generación eléctrica". VIII Congreso Nacional y 2do Congreso Internacional de Investigación. UC, (2013).

[51] Sandoval-Ruiz, C., and Fedón-Rovira, A. : "CESRCodificador RS $(255, \mathrm{k})$ eficiente para sistemas reconfigurables". Rev. Téc. Ing. Univ. Zulia, Vol. 37, N 2, (2014) 151-160.

[52] Sandoval-Ruiz, C. : "Arquitectura Reconfigurable y Redes Inteligentes aplicadas al Diseño Sostenible en Smart City". REC Perspectiva, Vol. 7, N 12, (2018) 8-21.

[53] Machado, Nelson Andrés López, et al. : "Evaluación del impacto de un techo verde sobre la escorrentía urbana usando un modelo a escala". Rev. Téc. Ing. Univ. Zulia, Vol. 43, (2020) 26-35. 


\section{REVISTA TECNICA}

DE LA FACULTAD DE INGENIERIA

UNIVERSIDAD DEL ZULIA

Vol. 43. $\mathrm{N}^{\circ} 3$, Septiembre - Diciembre 2020 pp. 114 - 176

Esta revista fue editada en formato digital y publicada el 31 de Agosto de 2020, por el Fondo Editorial Serbiluz, Universidad del Zulia. Maracaibo-Venezuela

www.luz.edu.ve

www.serbi.luz.edu.ve

www.produccioncientifica.org 